\title{
Memory effects in individual submicrometer ferromagnets
}

\author{
J. G. S. Lok, A. K. Geim, and J. C. Maan \\ High Field Magnet Laboratory, Research Institute for Materials, University of Nijmegen, NL-6525 ED Nijmegen, The Netherlands
}

S. V. Dubonos

Institute of Microelectronics Technology, 142432 Chernovolovka, Russia

\author{
L. Theil Kuhn and P. E. Lindelof \\ Orsted Laboratory, Niels Bohr Institute, Universitets parken 5, DK-2100 Copenhagen Ф, Denmark
}

(Received 9 February 1998)

\begin{abstract}
We have used ballistic Hall micromagnetometry to study the magnetization of individual submicrometer nickel disks (80 nm high, 0.1-1.0 $\mu \mathrm{m}$ diameter). At low temperatures, hysteresis loops of the disks no longer show inversion symmetry in a magnetic field, as if the time reversal symmetry were broken. Furthermore, the magnetization of the smallest disks can be "frozen" in two possible states that are characterized by hysteresis loops which are each other's inverse. At temperatures below $19.5 \mathrm{~K}$ a magnetic field as high as $2 \mathrm{~T}$ cannot switch between the states, proving that it is extremely difficult to fully polarize a small ferromagnetic particle. On the other hand, at slightly higher temperatures (only $T>19.8 \mathrm{~K}$ ), a field as low as $0.1 \mathrm{~T}$ appears to be enough to fully polarize the disks. We attribute this extraordinary behavior to the glass-liquid transition experienced by spins at the particle surface. [S0163-1829(98)06542-4]
\end{abstract}

\section{INTRODUCTION}

With the increasing need for high-storage-density media there has been an increasing research activity in the study of magnetization of small ferromagnetic particles. From a fundamental point of view measurements of the magnetization of an individual small particle are equally interesting, since they can provide on a microscopic level proof of the various theoretical models for magnetization reversal in larger, more complex systems. Newly developed techniques such as magnetic force microscopy, ${ }^{1}$ microsquids, ${ }^{2}$ and high-resolution near-field optical techniques ${ }^{3}$ have provided many interesting results on magnetization reversal in submicrometer ferromagnetic particles, ${ }^{4-6}$ among which are the experimental demonstration of coherent magnetization reversal in an ellipsoidal $\gamma-\mathrm{Fe}_{2} \mathrm{O}_{3}$ particle ${ }^{7}$ and experimental proof for NéelBrown thermally activated magnetization reversal in a single-domain particle with an activation volume equal to the particle volume. $^{8}$

We have developed the technique of ballistic Hall micromagnetometry, a noninvasive technique that can be used to study the magnetization of submicrometer ferromagnetic or superconducting particles at any temperature below $77 \mathrm{~K}{ }^{10}$ Here we present results on the magnetization of a set of submicrometer ferromagnetic nickel disks $(80 \mathrm{~nm}$ high, $0.1-1.0 \mu \mathrm{m}$ diameter). One of our most surprising results, which cannot be explained by current theories, is that at temperatures below $19.8 \mathrm{~K}$ the hysteresis loops of our nickel disks no longer show inversion symmetry, which seems a violation of time reversal symmetry. Furthermore, we observe that the magnetization of the smallest disks ( 0.1 and $0.2 \mu \mathrm{m}$ diameter) can be "frozen" into two possible states, neither of which show inversion symmetry. Instead, the hysteresis loop associated with the first state is the inverse of the hysteresis loop associated with the second state. At temperatures below $19.8 \mathrm{~K}$ these two states are very stable with respect to the magnetic field (i.e., application of a static magnetic field of $+10 \mathrm{~T}$ or $-10 \mathrm{~T}$ never flipped the magnetization from one state the other), showing that it is very hard to fully polarize the disks. At slightly higher temperatures, on the other hand, a field of only $+0.1 \mathrm{~T}$ or $-0.1 \mathrm{~T}$ fully polarizes the disks. The extremely sharp behavior versus temperature is suggestive of a phase transition, but the associated change in magnetization is smaller than $4 \times 10^{4} \mu_{B}$ (the sensitivity of our technique).

Our paper is organized as follows. First, we briefly describe the fabrication of our samples and magnetometer. Next, the principle of the operation of our magnetometer is explained. Finally, we present our measurements with a discussion of the experimental results.

\section{MAGNETOMETER AND SAMPLES}

Using a first round of lithography electrical contacts (NiAuGe) are defined onto a molecular beam epitaxially grown AlGaAs-GaAs heterostructure containing a twodimensional electron gas (2DEG) only $60 \mathrm{~nm}$ below its surface. Subsequently the contacts are annealed at $420^{\circ} \mathrm{C}$ for 5 min. Next, electron beam lithography is used to pattern a Hall bar into the PMMA (polymethyl methacralate) resist spun on the surface of the heterostructure. After developing the resist the Hall bar is etched onto the heterostructure using wet chemical etching in a 1:1:80 solution of $\mathrm{H}_{2} \mathrm{O}_{2}: \mathrm{NH}_{4}$ : $\mathrm{H}_{2} \mathrm{O}$. Finally, using a last round of electron beam lithography, the ferromagnetic material is evaporated over our structure and after lift-off only the material on top of the center of the Hall crosses remains. In this case the material is nickel and x-ray analysis of a thin film evaporated simultaneously with our samples shows that the material is polycrystalline. Initially samples were kept in a helium atmosphere but the results discussed below were not altered after aging the samples in air for 4 weeks.

Figure 1 shows a scanning electron microscopy (SEM) 


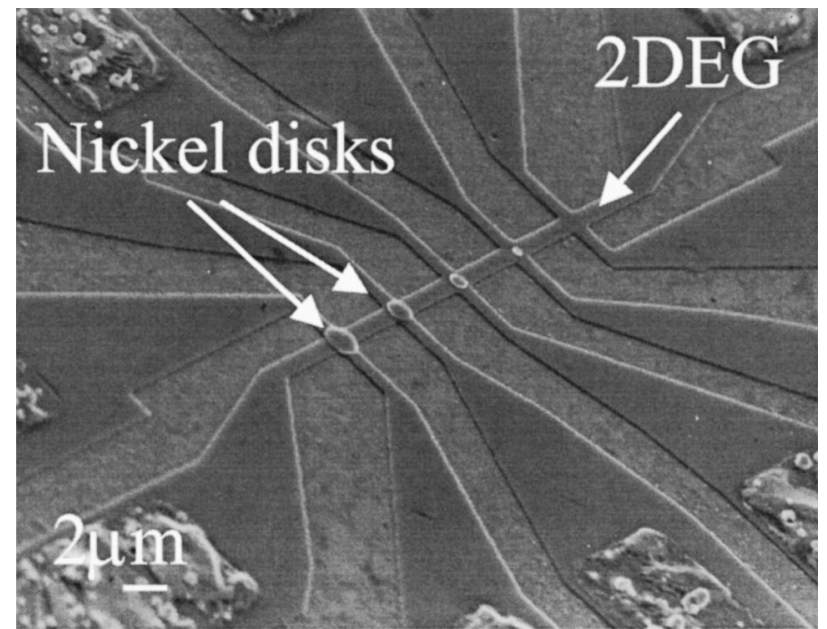

FIG. 1. SEM micrograph of one of our working devices. Nickel disks of various sizes appear as bright spots on top of the center of the Hall crosses. The micrograph is taken under an angle; in real life the side arms are perpendicular to the current-carrying wire.

micrograph of one of our working devices. It is a multiterminal wire with an effective width of only $1 \mu \mathrm{m}$. Nickel disks with a height of $80 \mathrm{~nm}$ and diameters ranging from 1.0 down to $0.1 \mu \mathrm{m}$ appear as bright spots on top of the center of the four most left Hall crosses. The height of the disks is monitored during evaporation with a film thickness meter (uncertainty 20\%) while the other dimensions of the disks and magnetometer are obtained from SEM micrographs (uncertainty $\sim 30 \mathrm{~nm}$ ). The disks are $5 \mu \mathrm{m}$ apart and thus interactions between them are negligible. Note that there is no electrical contact between the 2DEG channel and the nickel disks, because the $60 \mathrm{~nm}$ nonconducting GaAs cap layer provides a perfect electrical insulation. The outmost right cross is left empty to serve as a reference and as a sensor of the applied magnetic field.

\section{METHOD}

The principle of operation of our magnetometer is based on the fact that electrons moving in a magnetic field experience the Lorentz force and therefore a Hall voltage develops in the direction perpendicular to the current. This Hall voltage is easily detected using standard low-frequency lock-in techniques. The small ferromagnet, which is placed only 60 $\mathrm{nm}$ above the 2DEG, produces an additional magnetic field in the junction. As is the case for all near-field techniques, we do not measure the magnetization directly. It is the projection of the extra magnetic field caused by the ferromagnetic disk in the direction perpendicular to the 2DEG that is measured. We have used a 2DEG as a field sensor (and not an ordinary metal) because of its high mobility so that electrons move ballistically through the junction. For ballistic transport (unlike diffusive transport) the Hall voltage is proportional to the average magnetic field in the cross, ${ }^{9}$ and thus after subtracting the contribution to the signal of the applied magnetic field, the remaining signal is directly proportional to the magnetization of the disk, i.e.,

$$
\Delta R_{\text {Hall }}(\mathrm{G})=4 \pi M=\frac{1}{A} \iint_{A} B_{z, \text { ferro }} d x d y,
$$

with $A$ the area of the Hall cross and $B_{z, \text { ferro }}$ the z component of the magnetic field produced by the ferromagnetic disk. For the simple case of a magnetic field profile that below the disk area is equal to $4 \pi$ times the saturation magnetization $\left(M_{s a t}\right)$ and outside the disk area is equal to zero, the response of our magnetometer depends quadratically on the diameter of the disk $(d)$ and is given by $\Delta R_{\text {Hall }}$ $=(\alpha / A)\left(4 \pi M_{\text {sat }}\right) \pi d^{2}(\alpha=1)$. In reality, the flux lines close and the finite distance to the 2DEG needs to be taken into account. For uniformly magnetized disks placed $60 \mathrm{~nm}$ above the 2DEG, the functional dependence is still approximately quadratic (for diameters $<0.8 \times$ the width of the 2DEG channel), however with a numerical factor in front that corrects for the finite separation $(\alpha=0.049 \pm 0.004)$.

Finally, the large Hall coefficient of the 2DEG assures easily detectable signals. At liquid nitrogen temperature we have reached for our $1 \mu \mathrm{m}^{2}$ crosses a sensitivity that is limited by the Johnson noise which corresponds to $10^{-4} \Phi_{0}$ or less than $10^{4} \mu_{B}$. In order to circumvent significant degradation of the sensitivity at lower temperatures (arising from universal conductance fluctuations), we use a high excitation current (typically $3-5 \mu \mathrm{A}$ ) by which we heat up our electron gas to temperatures of about $20-30 \mathrm{~K}$. Note that this is the effective temperature of electrons in the 2DEG. We verified that the disks are not influenced by this high current and that over the entire temperature range studied $(0.3-77 \mathrm{~K})$ the disks remain at the set base temperature of our insert. We want to stress that this technique is completely noninvasive and for further details we refer to Ref. 10. The magnetic fields used in this study are produced either by a superconducting magnet or by an electromagnet, sweep rates range from 30 to $0.7(\mathrm{G} / \mathrm{s})$, and the measurements were performed with the magnetometer either at the end of a cold finger of a ${ }^{3} \mathrm{He}$ variable temperature insert or immersed directly into the ${ }^{3} \mathrm{He}$ liquid or gas (depending on temperature).

\section{RESULTS}

Figure 2 shows the hysteresis loops obtained for three nickel disks with diameters of, respectively, 400, 200, and $100 \mathrm{~nm}$ at liquid helium temperature after subtraction of the background that is due to the penetration of the applied magnetic field through the cross. The magnetic field is applied along the axis of the disk (i.e., perpendicular to the 2DEG) within $4^{\circ}$, but the results presented are not sensitive to the exact orientation of the magnetic field and are reproducible after remounting and thermal cycling to room temperature. The hysteresis loops presented in Fig. 2 are characterized by small jumps followed by a smooth increase of the magnetization in the region in between the jumps. The small jumps correspond either to a rearrangement of the domain structure in the particle ${ }^{4}$ or to the depinning of a single domain wall jumping from one local minimum (where it was pinned) to the next, while the smooth increase of the magnetization is due to the free movement of a domain wall through the crystal, in such a way that domains with a magnetization oriented in the direction of the magnetic field grow at the expense of less favorably oriented domains. The field over which the disks show coercivity increases with increasing diameter of the disk because of the increasing demagnetization factor and this is merely a magnetostatic effect. Furthermore, we 


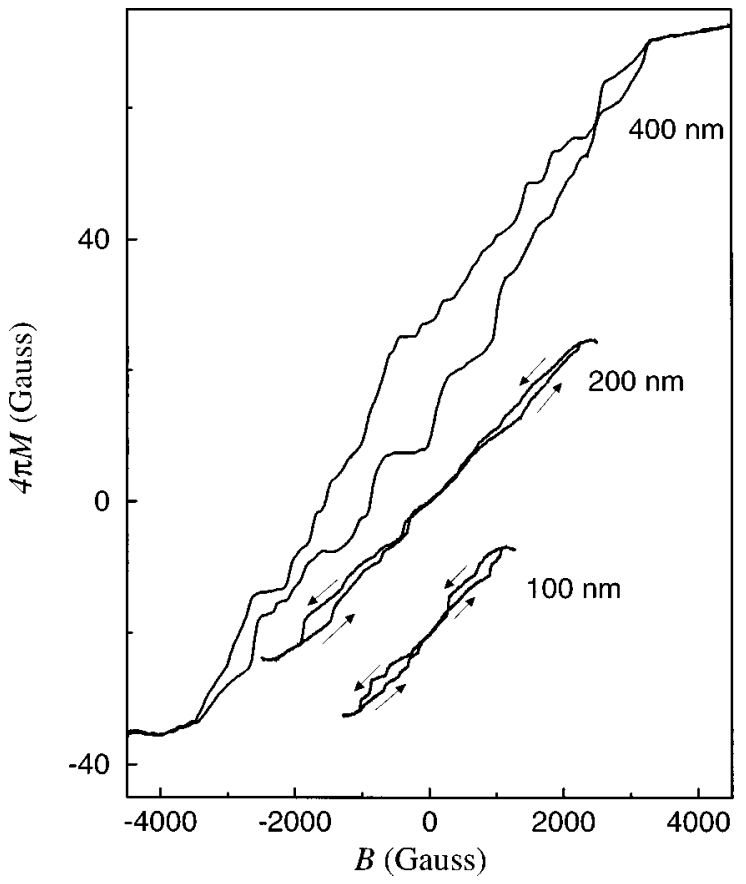

FIG. 2. Hysteresis loops for three nickel disks (80 nm high; 400, 200 , and $100 \mathrm{~nm}$ diameter, respectively) measured at $4.2 \mathrm{~K}$, clearly showing the absence of inversion symmetry. Curves for the $400 \mathrm{~nm}$ disk are offset, $+20 \mathrm{G}$; curves for the $100 \mathrm{~nm}$ disk are offset, $-20 \mathrm{G}$.

observe that the amplitude of the magnetization signal increases quadratically with increasing disk diameter. From a least squares fit to the experimental data with the saturation magnetization as the only fitting parameter, we deduce a value of the saturation magnetization of nickel of 430 $\pm 50 \mathrm{G}$, which is in good agreement with the value of $400 \mathrm{G}$ found in literature ${ }^{11}$ for unannealed nickel. The error in the saturation magnetization is partly due to the data scatter and partly due to the inaccuracy by which the various dimensions of the sample and the magnetometer are known.

The second observation is that the two smallest disks do not show any coercivity in the region around zero applied magnetic field. This probably indicates a flux-closure domain structure arrangement in these particles as was observed for thin, rectangular polycrystalline Permalloy particles in Ref. 4. For nickel disks with a diameter greater than $300 \mathrm{~nm}$ we always observe that the hysteresis loop in zero applied magnetic field has opened up, in agreement with previous investigations on small particles. 2,4

Figure 2 also presents our main result: the hysteresis loops of the three disks do not show inversion symmetry; i.e., when mirrored in the origin they do not map onto themselves. Even when we start from a very large positive field of $+10 \mathrm{~T}$ (which is $\sim 500$ times the anisotropy field of nickel and $\sim 20$ times the bulk saturation field) and sweep to a very large negative field of $-10 \mathrm{~T}$, the magnetization curve is not the same as that from a sweep in the opposite direction when mirrored in the origin. Instead exactly identical hysteresis loops as those presented in Fig. 2 are measured. This absence of inversion symmetry in the hysteresis loops is in obvious disagreement with the expected field reversal symmetry and it is only observed at low temperatures. At temperatures above $19.8-25 \mathrm{~K}$ hysteresis loops for the different

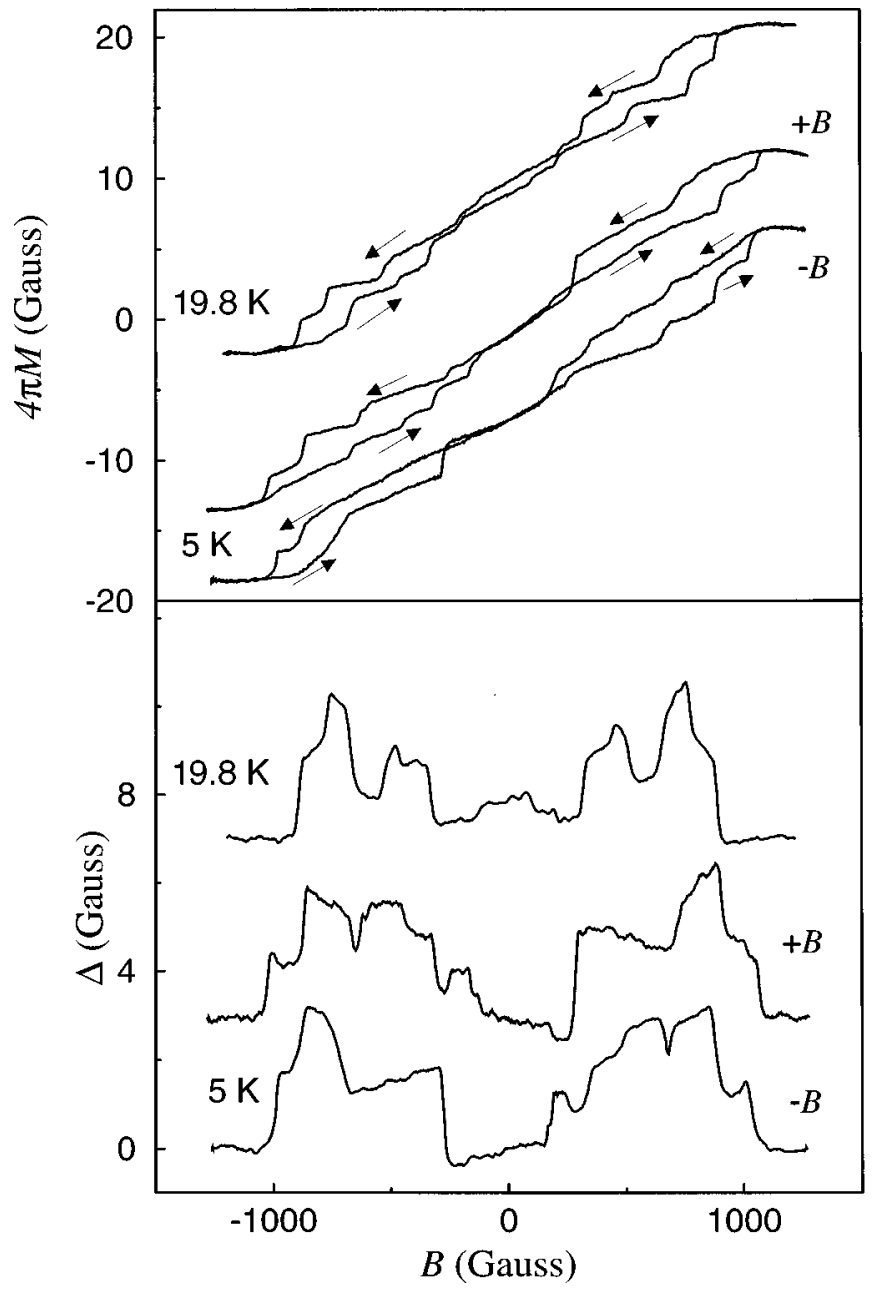

FIG. 3. Upper panel: hysteresis loops for the $100 \mathrm{~nm}$ nickel disk at $19.8 \mathrm{~K}$ (showing the expected inversion symmetry) and at $5 \mathrm{~K}$ after cooling in a positive field $(+\mathrm{B})$ and after cooling in a negative field $(-B)$. Lower panel: the difference in measured signal between a sweep up and a sweep down, to further illustrate that the hysteresis loops measured at $5 \mathrm{~K}$ are each other's inverse. (Curves are offset vertically for clarity.)

disks do show the expected inversion symmetry (see Fig. 3 top panel curve measured at $19.8 \mathrm{~K}$ ).

One could argue that since our crosses are not completely symmetric on a microscopic scale ${ }^{12}$ and since a Hall resistance measures an off-diagonal element of the OnsagerCasimir matrix, the Hall resistance is in general not identical when mirrored in the origin ${ }^{13}$ and that irregularities in the 2DEG arising at low temperatures cause the observed absence of inversion symmetry in the hysteresis loops. However, we verified that this is not the case by interchanging voltage and current contacts upon reversing the magnetic field polarity. For the empty crosses the Hall resistance measured at a positive field is equal to the Hall resistance measured at a negative field when current and voltage contacts are interchanged, while for crosses with disks on top we still measure the same hysteresis loops as those presented in Fig. $2 .^{14}$

Moreover, for the smallest disk we observe an even more drastic effect. At temperatures below $19.8 \mathrm{~K}$ the hysteresis loop for this disk no longer shows inversion symmetry. Instead, the magnetization of the disk is frozen in one of two 
possible states that are characterized by hysteresis loops that are each other's inverse. The top panel of Fig. 3 shows the hysteresis loops measured at $19.8 \mathrm{~K}$ and at $5 \mathrm{~K}$ after cooling (from $19.8 \mathrm{~K}$ ) in, respectively, a magnetic field of $+0.05 \mathrm{~T}$ and $-0.05 \mathrm{~T}$. The hysteresis loop measured at $19.8 \mathrm{~K}$ shows the expected inversion symmetry; i.e., when mirrored in the origin it maps onto itself (note that the curves in Fig. 3 are offset vertically). For either one of the two hysteresis loops measured at $5 \mathrm{~K}$ this is no longer the case. Furthermore, if we denote the hysteresis loop after cooling in a positive field by $M(B)$, the loop after cooling in a negative field is $-M(-B)$ and thus these hysteresis loops are each other's inverse. To further illustrate this inversion symmetry of the two possible magnetizations of this disk, the lower panel of Fig. 3 plots the difference between a sweep up and a sweep down. At $19.8 \mathrm{~K}$ this difference shows the expected symmetry with respect to inverting the magnetic field, while at $5 \mathrm{~K}$ neither the difference after cooling in a positive field nor the difference after cooling in a negative field displays this symmetry. Instead it is clearly seen that the difference after cooling in a positive field is the mirrored image of that obtained after cooling in a negative field. To quantify how well these hysteresis loops are each others inverse, we define a correlation factor $Q=\int\left|\left\{M_{+B}(B)-\left[-M_{-B}(-B)\right]\right\}\right| d B /$ $\int\left|\left\{M_{+B}(B)+\left[-M_{-B}(-B)\right]\right\}\right| d B$ with $Q=0$ for perfect agreement and $Q=1$ for no correlation. For both sweep directions we obtain $Q<0.03$. Furthermore, numerous cool downs from temperatures above $19.8 \mathrm{~K}$ in different magnetic fields (between $-10 \mathrm{~T}$ and $+10 \mathrm{~T}$ ) always lead to hysteresis loops that are either $M(B)$ or $-M(-B)$ (depending on the magnetic field polarity in the expected way). Also, at 16 $\mathrm{K}$ repeatedly applying a (static) magnetic field of + or $-10 \mathrm{~T}$ never flipped the magnetization of the disk from one state into the other, nor did a (static) field of + or $-2 \mathrm{~T}$ at $19.5 \mathrm{~K}$, while at $19.8 \mathrm{~K}$ the hysteresis loop has become inversion symmetric on applying a magnetic field as low as 0.1 T. Cooling from $19.8 \mathrm{~K}$ in a small positive (negative) field of only $+0.05 \mathrm{~T}(-0.05 \mathrm{~T})$ always flips the magnetization into the $+B$ state $(-B$ state).

For the $200 \mathrm{~nm}$ disk we observed the same effect (i.e., two possible hysteresis loops that are each other's inverse), although the temperature at which the hysteresis loop of this disk becomes inversion symmetric is slightly higher $(24 \mathrm{~K})$. Also for this disk it was not possible to use the magnetic field to change from one hysteresis loop to the other at temperatures below that at which the hysteresis loop shows inversion symmetry $(24 \mathrm{~K})$.

\section{DISCUSSION}

From the extraordinary behavior of the smallest nickel disk we can conclude that at temperatures below $19.5 \mathrm{~K}$ (16 $\mathrm{K})$ a magnetic field of $\pm 2 \mathrm{~T}( \pm 10 \mathrm{~T})$ does not destroy the "memory" of this disk, as it still "remembers" in which state it has frozen its magnetization. This unambiguously proves that at temperatures below $19.8 \mathrm{~K}$ the smallest disk is not fully polarized by applying these huge magnetic fields and thus that at $19.5 \mathrm{~K}(16 \mathrm{~K})$ the initial state in a magnetic field of $+2 \mathrm{~T}(+10 \mathrm{~T})$ is not the mirror image of the state in a magnetic field of $-2 \mathrm{~T}(-10 \mathrm{~T})$ (i.e., not all spins in the disk are reversed). In view of this remarkable result the above observed absence of inversion symmetry in the hysteresis loops of the disks at low temperatures is due to an incomplete polarization of the disks.

Furthermore, we can estimate from the temperature dependence an upper limit to the amount of magnetic moment with a spin opposite to the field direction by comparing thermal and magnetostatic energies. At $19.5 \mathrm{~K}$ an applied magnetic field of $2 \mathrm{~T}$ is not enough to overcome the barrier that separates the state of incomplete polarization from the state of complete polarization. On the other hand, a change in temperature as small as $0.3 \mathrm{~K}$ is enough to overcome this barrier. If the barrier itself does not depend on temperature, then the change in thermal energy $\left(k_{B} \Delta T=4.1 \times 10^{-24} \mathrm{~J}\right)$ is larger than the change in magnetostatic energy that is gained by applying a $2 \mathrm{~T}$ extra magnetic field $(M \cdot \Delta B)$. This poses an upper limit to the amount of antipolarized magnetic moment of only $0.22 \mu_{B}$. Similar reasoning for a $10 \mathrm{~T}$ extra applied magnetic field at $16 \mathrm{~K}$ leads to a slightly higher value of $0.6 \mu_{B}$. So far we assumed that internal and applied magnetic fields are equal. We note that a microscopically inhomogeneous distribution of the magnetic field near edges of the particle or pits in the particle surface may lead to considerable demagnetization fields. However, even when we assume that the local internal field at the position of the nonpolarized spins is only $1 \%$ of the applied magnetic field, still a magnetic moment of less than $22 \mu_{B}$ is involved. This corresponds to a reversed spin of less than 36 nickel atoms or a volume of reversed spins of less than $0.4 \mathrm{~nm}^{3}$.

Of course on this small length scale the above reasoning is too simplistic, since $M$ will not be uniform and since there will be a gradual change in the angle between successive spins farther away from the center of the antipolarized part. Nevertheless, we can exclude the possibility that so-called vestigial domains that have been observed in macroscopic samples near sharp corners or scratches on the surface and that persist in applied magnetic fields that are somewhat larger than the quoted saturation fields ${ }^{15}$ are responsible for the "memory" of our disks.

Instead the behavior versus temperature is suggestive of a phase transition (i.e., a temperature-dependent barrier). We therefore analyzed more carefully hysteresis loops measured after cooling in various positive and negative magnetic fields, yet under otherwise identical experimental conditions, but found that they overlap within our experimental resolution of $4 \times 10^{4} \mu_{B}$. Furthermore, temperature sweeps during which the temperature is slowly increased to above $19.8 \mathrm{~K}$ and which are measured in a constant applied magnetic field of $0.1 \mathrm{~T}$ after the sample had been cooled in + or $-2 \mathrm{~T}$ do not reveal any jump in magnetization within the noise level $\left[\left(5 \times 10^{3}\right) \mu_{B}\right]$, confirming that the amount of unpolarized magnetic moment is truly nanoscopic.

From experiments on collections of nanometer-sized particles ${ }^{16-18}$ it is known that field cooling can drastically alter the magnetic properties of collections of small particles. In particular, field cooling can result in shifted hysteresis loops or enhanced magnetic moments and this has been attributed to the behavior of spins located at the (possibly oxidized) surface of the particles that freeze below a certain temperature into a spin-glass-like layer in which their orientation is pinned along the direction of the magnetic field that was applied during cool down. A spin-glass-like phase tran- 


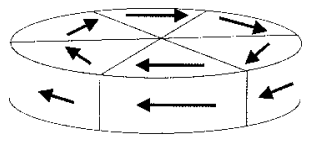

Clockwise

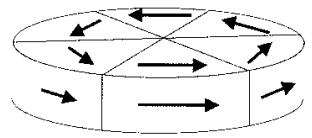

Counter clockwise
FIG. 4. Schematic drawing of the two possible flux-closure domain structure arrangements in the smallest disks in zero applied magnetic field. Arrows indicate the direction of the magnetization in the domains.

sition can explain the fact that for the different particles studied, the hysteresis loop becomes symmetric at slightly different temperatures (between 19.8 and $25 \mathrm{~K}$ ). Also, in a disordered oxidized surface layer, canted spins serve as nucleation centers for domain walls and this explains different hysteresis loops after field cooling. On the other hand, we note that if the surface of our $100 \mathrm{~nm}$ disk were atomically flat (which it certainly is not), still $1.3 \%$ of the atoms would be at the particle surface which corresponds to a magnetic moment of about $\left(4 \times 10^{5}\right) \mu_{B}$. Considering the abovequoted detection limit, this would imply that at most only $10 \%$ of the total magnetic moment carried by the surface spins is frozen in the spin glass.

Finally, we want to comment on the remarkable observation of two possible hysteresis loops in one particle that are each other's inverse. As stated above this effect is observed only for the smallest disks. The hysteresis loop of these disks does not show coercivity in zero applied magnetic field and this points to a flux-closure domain structure in the particle schematically sketched in Fig. 4. By arranging domains in a more or less head-to-tail alignment in the plane of the disk, the particle avoids free poles at its surface and thus minimizes its free energy. At high temperatures when the hysteresis loop shows field reversal symmetry, if in zero applied magnetic field this flux-closure domain structure is clockwise and we sweep the magnetic field down to zero after applying a "saturation" field of +0.1 or $+0.25 \mathrm{~T}$, the counterclockwise flux-closure domain structure appears. After applying a saturation field of -0.1 or $-0.25 \mathrm{~T}$, again in zero field the clockwise flux-closure domain structure appears. On the other hand, at low temperatures when the hysteresis loop no longer shows field reversal symmetry, we believe the "frozen" surface spins to serve as nucleation centers for domain walls in such a manner that after cooling in a positive field, both on sweeping the magnetic field up through zero as well as down through zero, the clockwise flux-closure domain structure appears, while after cooling in a negative magnetic field, always the counterclockwise flux-closure domain structure is present in zero applied magnetic field (or vice versa of course). So the pinning mechanism active in the particle at low temperatures breaks the field reversal symmetry, but on application of a positive magnetic field to the clockwise fluxclosure domain structure exactly the same domain wall movements or annihilations will occur as on application of a negative magnetic field to the counterclockwise flux-closure domain structure and thus part of the symmetry is recovered. Provided there is a strong pinning mechanism, the model described above naturally explains the observation of two possible hysteresis loops in one particle that are each others inverse.

\section{CONCLUSIONS}

In conclusion we have investigated the magnetization of individual submicrometer polycrystalline nickel disks using the technique of ballistic Hall micromagnetometry. At low temperatures $(T<\sim 20 \mathrm{~K})$ we observed that the hysteresis loops of the nickel disks no longer show inversion symmetry. Furthermore, we showed for the two smallest disks investigated the existence of two possible states of the magnetization (within one disk) which are characterized by hysteresis loops that are each other's inverse. An applied magnetic field of $10 \mathrm{~T}$ never flipped the magnetization from one state into the other, proving that it is very difficult to fully polarize the disks at low temperatures. The pinning of the magnetization in these disks shows a behavior versus temperature and magnetic field that is suggestive of a phase transition experienced by spins at the particle surface.

\section{ACKNOWLEDGMENTS}

This work is part of a research program of the Stichting voor Fundamenteel Onderzoek der Materie (FOM) financially supported by NWO (The Netherlands).
${ }^{1}$ For a review on magnetic force microscopy, see J. A. Sidles, J. L. Garbini, K. J. Bruland, D. Rugar, O. Züger, S. Hoen, and C. S. Yannoni, Rev. Mod. Phys. 67, 249 (1995).

${ }^{2}$ W. Wernsdorfer, K. Hasselbach, D. Mailly, B. Barbara, A. Benoit, L. Thomas, and G. Suran, J. Magn. Magn. Mater. 145, 33 (1995).

${ }^{3}$ For a review on near-field optics, see D. W. Pohl, in Scanning Tunneling Microscopy II, edited by R. Wiesendanger and H.-J. Günterodt, Springer Series in Surface Science Vol. 28 (SpringerVerlag, Berlin, 1992), p. 233.

${ }^{4}$ S. J. Hefferman, J. N. Chapman, and S. McVitie, J. Magn. Magn. Mater. 95, 76 (1991).

${ }^{5}$ M. Lederman, G. A. Gibson, and S. Schultz, Phys. Rev. Lett. 73, 1986 (1994).

${ }^{6}$ W. Wernsdorfer, B. Doudin, D. Mailly, K. Hasselbach, A. Benoit,
J. Meier, J.-Ph. Ansermet, and B. Barbara, Phys. Rev. Lett. 77, 1873 (1996).

${ }^{7}$ C. Salling, R. O-barr, S. Schultz, I. McFadyen, and M. Ozaki, J. Appl. Phys. 75, 7989 (1994).

${ }^{8}$ W. Wernsdorfer, E. Bonet Orozco, K. Hasselbach, A. Benoit, B. Barbara, N. Demoney, A. Loiseau, H. Pascard, and D. Mailly, Phys. Rev. Lett. 78, 1791 (1997).

${ }^{9}$ X. Q. Li, F. M. Peeters, and A. K. Geim, J. Phys.: Condens. Matter 9, 8065 (1997).

${ }^{10}$ A. K. Geim, S. V. Dubonos, J. G. S. Lok, I. V. Grigorieva, J. C. Maan, L. Theil Hansen, and P. E. Lindelof, Appl. Phys. Lett. 71, 2379 (1997).

${ }^{11}$ Handbook of Chemistry and Physics, edited by D. R. Lide (CRC Press, Boston, 1992).

${ }^{12}$ In zero applied magnetic field the ratio of the diagonal to longi- 
tudinal resistance for the empty crosses is smaller than 0.01 (but not zero).

${ }^{13}$ M. Büttiker, Phys. Rev. Lett. 57, 1761 (1986).

${ }^{14}$ For some of our Hall crosses the sensitivity of our magnetometer differs slightly upon changing magnetic field polarity: see, for instance, Fig. 3, curve measured at $19.8 \mathrm{~K}$, where a close examination of the data reveals a slightly larger signal for positive magnetic fields. When current and voltage contacts are interchanged the signal is larger for negative magnetic fields; so this effect is due to a slight asymmetry in the exact geometry of the Hall cross.

${ }^{15}$ U. Hartmann, Phys. Rev. B 36, 2331 (1987).

${ }^{16}$ R. H. Kodama, A. E. Berkowitz, E. J. McNiff, Jr. and S. Foner, Phys. Rev. Lett. 77, 394 (1996).

${ }^{17}$ R. H. Kodama, S. A. Makhlouf, and A. E. Berkowitz, Phys. Rev. Lett. 79, 1393 (1997).

${ }^{18}$ B. Martinez, X. Obradors, Ll. Balcells, A. Rouanet, and C. Monty, Phys. Rev. Lett. 80, 181 (1998). 